\title{
ABIOGENESIS TRANSITION FROM THE ATMOSPHERE INTO THE HYDROSPHERE: FROM VESICLES TO PROTOCELLS
}

\author{
Alexander R. Zaritsky \\ Lebedev Physics Institute, Russian Academy of Sciences, http://www.lebedev.ru \\ 119991 Moscow, Russian Federation \\ zaritsky@sci.lebedev.ru
}

\section{Vladimir I. Grachev}

Kotel'nikov Institute of Radio Engineering and Electronics, Russian Academy of Sciences, http://www.cplire.ru 11/7, Mokhovaya str., 125009 Moscow, Russian Federation

grachev@cplire.ru

\section{Yuri P. Vorontsov}

Filatov Hospital no. 13, Moscow Department of Healthcare, http:/ www.13dgkb.ru

103001 Moscow, Russian Federation

yupmkpsp@rambler.ru

\section{Vyacheslav S. Pronin}

Sechenov First Moscow State Medical University, Ministry of Healthcare and Social Development of RF, http://www.mmm.ru 119991 Moscow, Russian Federation

vspronin@yandex.ru

\begin{abstract}
Presented probable variant of stage transition of abiogenesis from the atmosphere into the hydrosphere - the transition of abiogenic organics from planetary autoclave of near-surface oxygen-free atmosphere into hydrosphere of a cooling planet and development of abiogenesis in the hydrosphere before its completion with the emergence of protocells. Herewith, the hydrocarbon droplets of atmospheric aerosol - the main sources of energy of formation of organic matter in the primitive atmosphere, when dissolved in an turbulent aqueous medium transformed into vesicles - centres of development of primitive energy metabolism and flowing homeostasis, inherited from drops. Interaction of viral world, which appeared in the atmospheric period of abiogenesis, with the world of lipid vesicles ensured the development in them of mechanisms initially anaerobic and then aerobic external and internal energy metabolism. Herewith an important role in the metabolism of vesicles played the selection compounds by type of symmetry. This selection narrowed the range of compounds involved in the internal metabolism of vesicles and provide optimal occurrence of appropriate mechanisms of developments of nascent cellular world
\end{abstract}

Keywords: abiogenesis, atmosphere, hydrocarbon nanodroplets, autonomous energy source, metabolism, homeostasis, primary separation, lipids, vesicles, viruses, molecular symmetry.

PACS: 87.23.Kg

Bibliography - 11 references

Received 28.11.2014

DOI: 10.17725/RENSITe.0006.201412f.0221

\section{CONTENTS}

1. INTRODUCTION (221)

2. «ACTIVE» LIPID FILMS AND VESICLES (222)

3. Metabolism open SyStems as THE PREDECESSOR OF THE PROTOCELLS ACTIVITY AND FISSION (223)

4. EVOLUTION OF THE WORLD VESICLES, APPEARANCE OF PRECURSOR CELLS AND THEIR ORGANELLS (225)

5. SElection OF COMPOUNDS BY THE TYPE OF SYMMETRY (225)

6. AppeARANCE OF THE FIRST PROTOCELls (226)

7. Final stage of abiogenesis (228)

8. ConClusion (229)

REFERENCES (230)

\section{INTRODUCTION}

The subject of this paper is the abiogenesis final stage, start of which is the transition of the "prelife" from the atmosphere into hydrosphere of the planet when it cools, finish is the first appearance of the archaic cells. Prior to that stage the germs of life have gone a long way of the evolutionary development from nonequilibrium aerosol organic systems - the hydrocarbon nanodroplets [1] - the first sustainable confined sources of free energy in the first subvital zone of the planet - in the planetary autoclave of 
near-surface oxygen-free atmosphere, to form a sufficiently rich set of structures and mechanisms "prelife". Along with organics captured during accretion of the planet from the protoplanetary cloud appeared and developed own organics, conditions of forming of which seem to be quite sufficient. This conditions are the temperature and the required values pressure, saturating of near-ground layers of the atmosphere with water vapor and minerals, the turbulence of these layers, energy of natural sources - Sun, heat, radioactivity and, finally, autonomous sources - world of hydrocarbon drips that ensures abiogenesis by energy. The initial stage of abiogenesis in oxygenfree atmosphere with a predominance of methane, carbon monoxide and ammonia in the near-surface dense dust layer with vapors and suspensions of minerals - natural catalysts is characterized the synthesis of the simplest hydrocarbons. This are amino acids up to the chain peptides and ribozymes (first organic catalysts), nucleotide and their chains up to RNA polynucleotides, lipids, archaic macroergs finally viruses. Was formed mechanisms of primitive metabolism - the exchange of matter and energy with the environment and the flowing homeostasis - maintaining the stability of the elements and variables (temperature, $\mathrm{pH}$, etc.) in the flowing interaction with the environment. When condensation with water vapor, this organics passed into the aquatic environment of the formed hydrosphere planet.

If at the beginning of evolutionary transformations of such systems polynucleotides are only used as a depot (warehouse) archaic macroergs, then by the time of the transition abiogenic organics into the aquatic environment have developed mechanisms of matrix reproduction of complex compounds, including polynucleotides themselves in multicomponent reactions. Significant, apparently crucial role in these mechanisms played (and play up to the present time in cells) lipids light (several hundred Daltons), linear molecules of relatively small size (about $1 \mathrm{~nm}$ ), synthesized on hydrocarbon droplets and to partially or completely covered them surface. Flowing homeostasis of lipid massa provides the convergence to it of all the reactants into the reaction zone. Processes of lipid metabolism have lead to a shift of the lipid mass together with the her associates and their complexes to a convoluted chains of amino acids - to the primitive proteins - enzymes, that perform catalysis of decomposition reactions energy-intensive lipid [2]. The energy of this decay can be used in the synthesis reactions of other compounds, including archaic polynucleotides and macroergs. On this basis, on the surface of lipids began to pile up and operate mechanisms of forming organelles (plastids, vacuoles, ribosomes, vesicles, tubular structures, etc.), that provide an interface of reactions of the matrix reproduction of complex compounds and replication of polynucleotides.

The pinnacle of the evolution development of atmospheric phase "prelife" was the appearance of the first virus-like bodies and, finally, of primitive viruses [2]. Combinatorics of peptide compounds, feedbacks between the matrix reproduction and replication of primary information polynucleotides in a symbiotic relationship with local sources of free energy, that utilize the stocks of atmospheric hydrocarbonic raw, all of this made an emergence of viruses at this stage inevitable. Because metabolism drops was open, their surface structures - the compounds and their complexes, including with polynucleotides, were able to as go to the gas medium well as come back at the drops surface, providing reproduction of compounds. For rapid evolutionary development of the first viruses in the gaseous medium formed the ideal conditions: the open metabolism of drops have guaranteed the energy basis, and frequent mutations of the information parts in an aggressive and highly turbulent medium of the then atmosphere provide a flow of new information.

\section{2. "ACTIVE" LIPID FILMS AND VESICLES}

The result of the transition of the atmospheric organics into the aquatic environment, which appeared on the cooling surface of planet by condensation of water vapor (along with endogenous - mantle [3] and exogenous - meteorites [4] and ice rains [5] - the sources of primary hydrosphere), became "a primary soup ", whereof wrote A.I. Oparin [6]. Autonomous sources of free energy - "active" hydrocarbon droplets, archaic enzymes and lipids on their surfaces at the transition from atmosphere to aqueous medium completely or partially lost of their lipidic shells, as schematically shown in Fig. 1. 


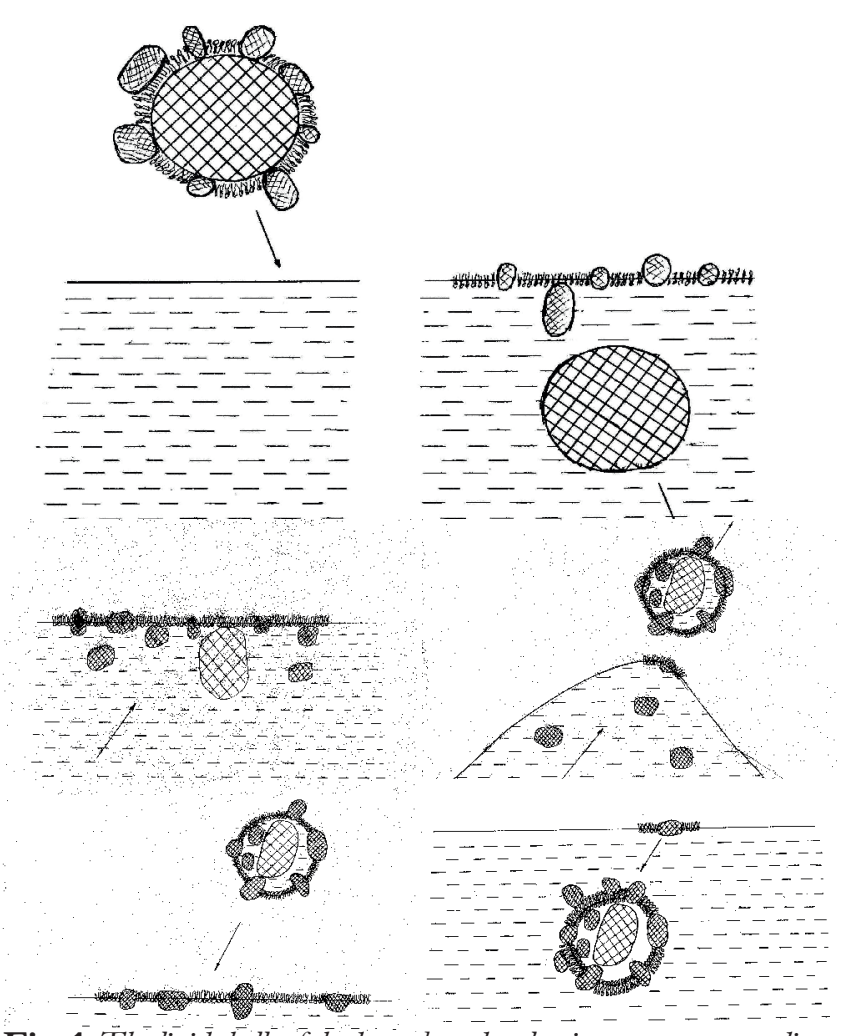

Fig. 1. The lipid shells of bydrocarbon droplets in an aqueous medium.

On the water surface are the single-layer lipid films with embedded and their associated archaic enzymes. Due to the strong turbulence at the phase boundary between atmosphere and water surface of these films were formed vesicles ${ }^{1}$, just as it is done in laboratories by sonication. Some of the films and vesicles in the new conditions of water medium have inherited from hydrocarbon droplets the mechanisms of metabolism. It is important to note that the metabolism of vesicles could be both external and internal, and some of them had got and that and other at the same time. In turn, only some of the viruses are able to interact with these structures in the new environment, more dense than water vapor. For emerging living world this situation can be described as a catastrophe, bifurcation. Compared to the lifetime of our Galaxy the transition time "prelife" in the aquatic environment looks as a moment, and compared with the appearance and development of our civilization - almost an eternity.

Abiogenesis had continued to evolve in the new environment. The lipid films on the aqueous surface and the lipid vesicles in thicker of aqueous medium "replaced" as sources of free energy the

\footnotetext{
${ }^{1}$ Vesicle - cavity bubble (lat. vesica - bubble) to $100 \mathrm{~nm}$ in diameter, in a lipid shell with an aqueous solution of nucleotides.
}

hydrocarbon droplets atmospheric aerosol. The carbon-containing compounds energy of decay on the surface of films and vesicles provides a synthesis of all of the abiogenic organics - polynucleotides, archaic viruses, enzymes and macroergs. Hydrolysis of macroergs largely complemented the energy for synthesis of compounds involved in flow metabolism of open systems in an aqueous medium.

Note that such anoxic synthesis of macroergs in conjunction with enzymatic disintegration of compounds (including enzymes themselves), independent of presence of macroergs in reaction zone, is used and at present, not only in red blood cells, but also and in all cells in quiescent. The presence of such ways of obtaining macroergs in addition to the main energy-dependent processes for their synthesis, provides the cells in quiescent the concentration stability of these compounds, including and ATP.

\section{METABOLISM OPEN SYSTEMS AS THE PREDECESSOR OF THE PROTOCELLS ACTIVITY AND FISSION}

The question of occurrence in the cells mechanisms of their fission seems unsolvable if to approach this problem under the assumption that these mechanisms division formed in the cells themselves and, most importantly, for them. The situation is different if we assume that these mechanisms began to take shape for the reproduction of viruses in the period of abiogenesis in an aqueous medium, and then inherited by cells for mitotic activity.

Abiogenesis in the gas and aqueous medium lasted parallel many millions (if not billions) of years under the leading (in the transformation of organic matter) role of viruses, that evolving in atmosphere more quickly. Was formed new feedbacks between viruses of both media and local sources of free energy. In the aqueous environment surrounding the energy sources (lipid vesicles and films), and in vesicles appeared a sufficient quantity of macroergs for replication of information pieces and bodies viruses.

Alongside with the concentration macroergs non equally important factor is to maintain in the aqueous medium the reaction zone of acidity, optimal for multicomponent reactions metabolism. Intensification of oxidative processes in which 
used oxygen of water molecules inevitably led to acidification of reaction zone, changing the rate of metabolic reactions, reducing the power of sources. In process of the aqueous medium acidification the reaction zone was shifted further away from the surface of the lipid films and vesicles, where are mainly generate free energy with the release of ions $\mathrm{H}+$, contributing to the primary reactions of rapid synthesis of the biologically active small compounds, for example, short peptides, whose the non-ribosomal synthesis can be carried out quickly and does not require high energy expenditure [7]. Such compounds known and currently, and molecular mechanisms act in the present-day cells and in the internal environment of multicellular organisms.

Implementation of these ways to improve molecular mechanisms metabolism and of strengthening on this basis of energy generation led to the emergence of new structures that could later be used after the upgrade in cells and for other purposes. So, the chains reactions alkalizing of cytoplasm were needed for the organization of a cyclic mode of activity cell [8] and the complex oscillating regimes of acidification and alkalization in the background of natural acidification of the cytoplasm during mitosis [9].

Let us explain the last statement. Since modern complex processes of cellular metabolism requires energy, then, if there is competition between the two major groups of primary energy-dependent processes for macroerg ATP (meaning the group that involved in the synthesis of ATP and the group that in this is not involved) the maximum possible efficiency work of each can be achieved by disseminate their action in time. Herewith the systemically important factors are typically the changes of acidity and concentration macroergs. Basic methods and means of implementing such a time diversity of processes are mentioned above. So in the activity mode of modern cells are for this used the cyclic changes of $\mathrm{pH}$ and concentrations of the main macroerg in the cytoplasm [8]. At mitosis the changes of this parameter have more complex character, however, achieved by the same means. With the accumulation in the depot of energy-consuming and plastically material, enough to power a few cell divisions, a signal is generated to start mobilizing of depot material. In parallel, this same signal generates the increased of alkalizing processes of cytoplasmic before neutral values, causing the cell "reminisces" the condition of their first evolutionary predecessors. To the extent of uptake of energy-intensive substrates the acidification of the cytoplasm naturally grows from neutral values. Precisely this determines the sequence of switching and completing all the processes of fission [9]. Molecular mechanisms of automatic activation and regulation of alkalizing and acidifying the cytoplasm during mitosis are formed ("are grow") in considered evolutionary period out of archaic cycle of the precursor cells metabolism. So cyclic changes the length of fibers spindle, caused by the change dominance of processes of their growth and decay, as well as the change of value affinity chromosomes to their ends of the at the equator, defining processes of the "shake-up" and "recognition" by chromosomes "own" thread can be explained by the superposition of the archaic cycle on mitotic cycle in phase of the monotonic rise acidification of the cytoplasm during mitosis [9].

We should also mention the possibility of appearing in the period under review another phenomenon: the emergence of the tubular structures of lipids, which departs from the surface of vesicles into surrounding aqueous environment. Such structures are could to appear in the case of temporary dominance of lipids synthesis over their decay as an macroergs by the steady intensification of energy metabolism and $\mathrm{pH}$ fluctuations of adjacent aquatic environment. Since the replication of information parts of viruses occurred also due to the decay energy of lipids, their indicated tubular structures could be used as a depot of this peculiar macroerg.

In concluding this section, we note that the level of acidity and concentration of macroergs in the aquatic environment and their natural variations in open systems that generate energy, in the period under review proved to be useful for the reproduction of increasingly sophisticated viruses. Accordingly, appeared new tools and molecular mechanisms that have ensured for this the optimum conditions and have given a start to the formation of the mitotic apparatus and means of cyclic acidification-alkalinization modes of processes the cell metabolism. The formation 
of such mechanisms began at the considered abiogenesis stage in the processes of reproduction and evolutionary development of viral world, long before the appearance of first cells and the final formation of the living world

\section{EVOLUTION OF THE WORLD VESICLES, APPEARANCE OF PRECURSOR CELLS AND THEIR ORGANELLS}

A previous sections have been devoted to the consideration of evolutionary development of external metabolism of lipidic films and vesicles. His openness for interaction with viruses ensures rapid development of the viral world and, accordingly, equally rapid complication of metabolic structures that provide energetic support of evolutionary changes. Behind the scenes is still the fate of structures that "are trapped" into the vesicles. We emphasize "trapped", because the lipid films and vesicles of that time were by dynamic structures. Recall that the organics exchange of aqueous medium with the atmosphere was continued, although its intensity gradually was falling. From films were appearing new vesicles. They were seizing the organics, the source of which - a films which were broking by the shear flows. And the "membranes" of vesicles, who died under the dominance of decay of lipids (the analog cell death by way of necrosis). Vesicles could be combined into larger individuals in random collisions and merging. Their contents were combined. The internal environment of vesicles have enriched with new structural elements, useful for her metabolism. This happened in the flow (replacement, substitution) of composition vesicle. Thus at indirect interacting with viruses, internal metabolism of vesicles could develop. However, the criteria for peculiar selecting and securing of new information (in according to EM Galimov new compounds [10]) were slightly different than for external metabolism. External metabolism developed in cooperation with viruses. This interaction was the leading factor in this development. Development of external metabolism was progressive, with the proviso that the development of virus was also progressive and the representation of viruses was increased in nature. Outdoor metabolism provided the viruses the pick new information. Internal metabolism was developed using structures that confer resistance to the harmful effects of turbulent water environment. These two processes were parallel. This interaction of two metabolic systems with each other and with the viruses prepared and implemented the preconditions for the emergence of the first precursors of organelles and cells.

Indeed, the vesicles with their primitive outer and inner metabolism remind us the mitochondria, which act in oxygen-free environment of the primitive ocean, the whole mass of water which can be identified with "the cytoplasm" planetary scale. Isolation of metabolic structures was casual, but widespread. This determined the inevitable emergence of bubbles with a lipid envelope, which from the incomplete enzymatic pool is not capable of generating energy at its outer side. Among them were a vesicles, whose internal metabolism can provide the energy of the replication of captured polynucleotides and the reproduction of other compounds. Such vesicles with good reason can be attributed to the ancestors of the first cells, and a vesicles that have external and internal metabolism, can be attributed to their predecessors of energy organelles.

\section{SELECTION OF COMPOUNDS BY THE TYPE OF SYMMETRY}

One of the most characteristic processes inherent to abiogenesis - the vesicle contents formation by selection available having at that time of organic compounds by the chirality - the type of symmetry of the molecules with respect to their optical activity (right or leftrotatory the plane of polarization of light). All biota is chiral, unlike nonbiological organics. For instance, all the amino acids can be in relation to the plane of polarization of the incident light only the left rotatory (and exactly they are the parts of protein), and sugar - only dextrorotatory (the parts of RNA and DNA). Questions, how they have been selected and whether it contributed to the emergence of the first cells remain unanswered.

One option of answer is possible in the context of the necessary "benefit" for the nascent cellular world. Selection entering into the vesicles compounds by the type of symmetry substantially has reduced the time (had to select from a much smaller number of elements) in the formation in the vesicles the 
structures and mechanisms that distinguish the first cells from its predecessors.

Possible following mechanism of this selection. Chiral element macromolecule (usually an asymmetric carbon atom is) influences the structure of the electron density of its atoms so that the dipole moment (multipole of first-order) molecule is oriented in the direction of its low electron density, i.e. the minimum of the cross-sectional of linear molecule. Such a molecule in the field membrane, directed inwardly of vesicle, too is orienting her dipole moment to inside, and encounters a little resistance to the her small cross-section. Whereas for the achiral molecule her dipole moment (if any) is not orientated oneself toward the low electron density, i.e. the minimum cross-sectional area of the molecule. And resistance to her movement in the field membrane is insurmountable.

For example, a chirally dextrorotatory D-glucose (as deployed in a linear conformation) has a dipole moment that faces to the carbonyl positive end with a minimum electron density i.e. with a minimum cross-section. While on her negative pole the group $\mathrm{CH} 2 \mathrm{OH}$ oriented perpendicular to the axis of the molecule, ie, here her cross-section is maximized. But in membrane her field orients the "acute" end the glucose molecule inward vesicle. The resistance to her movement inward is minimum, and the membrane is permeable to such a molecule. In experiments with whole blood and erythrocytic mass, dilute by physical solution, previously ${ }^{2}$ we have shown that D-glucose accumulates in the cytoplasm, and the larger, more the potential difference set at erythrocyte membranes.

Likewise occurred, apparently the filling of vesicle contents with compounds of defined chirality, i.e. selection of compounds by the type of symmetry.

\section{APPEARANCE OF THE FIRST PROTOCELLS}

We have shown above, in the period under review are ripe conditions for the emergence of the first cells. Their predecessors - "active" vesicles already possessed primitive genome and energy metabolism that can provide energy not only for replicate their data connections, but also for the reproduction of most of the compounds of their

${ }^{2}$ In 80-90th years these experiments were initiated and started by Andrei Agasievich Charakhch'yan (FIAN USSR). internal environment. In the selection process their resistance to aggressive environmental influences increased. A cyclic metabolism (archaic cycle) was forming, which ensure the achievement of their maximum capacity with help of the alternating interchange of energy-dependent processes. Herewith a leading backbone factor of innovation action in vesicles (other than energetics) became the acidity of their internal environment. With regard to the membrane vesicles, balance velocities of synthesis and degradation (expenditure) of their lipids observed only accidental and for a short time. Further was following inevitably the ruin of vesicle through the path to necrosis or apoptosis. This fact was accelerating the testing of individual vesicles on the stability at the initial stage of the cell world forming. It was rather helpful than harmful for the evolutionary transformations. However, with the growth of energy metabolism capacity, such mismatching of the velocities of synthesis and breakdown of lipids became brake for evolutionary development. Intensification of deposition of lipidic mass outside and inside the vesicles, including in the form of tubular structures, additional membranes and other structures that include lipids, was reducing significantly the probability of their ruin through the path to necrosis or apoptosis. To this also contributed the emergence of the depositing of energy-intensive substrate, which assimilated inside the vesicles, and was the most represented in the environment. Innovations largely decreased danger of a ruin of vesicles. In addition, some types of such structures of depositing became fulfil protective function. This also strengthens stability of vesicles to destructive influence of environment.

In the final stages of becoming of cellular world, such depots were ready to provide energy and materials for mitotic activity. However, the progressive movement of elements of the arising cells world towards the increasing complexity of their structures, and first of all information parts was starting significantly hindered. The fact that the intensification of the internal energetic metabolism (general line of development of living world) inevitably led to an increasing acidification of the internal environment of the vesicles. A situation shaped, similar to the one that was discussed above at competition for energy of two groups 
of metabolism processes. At this time we are talking about competition for energy of replication processes increasingly complex information molecules and processes of reproduction of all other compounds involved in the metabolism [11]. Recall that this is the end result of interaction of open, and then internal metabolism of vesicles with viruses. Upon acidification of the medium compared with the waters of the archaic ocean the viral bodies lose affinity for information parts, freeing them for their replication and their reproduction at the expense of energy of external metabolism. The internal environment of the vesicles with a functioning energy metabolism was always been acidified. Therefore, at the initial stage of evolution constraints for reproduction genetic material within such vesicles was not. At the beginning, information molecules replicate by a relatively weak acidification of the internal environment, and then, at a significant acidification starts reproduction of connections of enzymatic base. Such a procedure has found its consolidation in the archaic cycle. However, as the evolutionary development of energy metabolism, which characterized by further intensification of oxidative processes, the increasing complexity of the genome and the growth of information pieces to be replicated, more rapid acidification of the internal environment prevented the completion of replication. In addition, the archaic cycle acidification-alkalinization increasingly shifted to the acidic side, exacerbating competition for energy in reproduction of connections between the genome and the pool of enzymes that were in the past a viral bodies. Separation of archaic cycle into the cycle metabolic activity and mitotic cycle has removed this difficulty. This became possible after the emergence of new means of regulating the activity of enzymes that serve the depot of the plastic and energy-intensive materials, and enzymes responsible for alkalinization of internal environment of vesicles. With the growth of depot and accumulation material therein, sufficient to allow the mitotic activity, using these new means was generated a signal, which provided, firstly, a rapid and deep (to neutral $\mathrm{pH}$ indicator values) alkalinization of internal environment of vesicles and secondly, the start of mobilization with subsequent utilization of the material depot. Herewith the system "reminisces" the traversed evolutionary path by reproducing its major milestones (Haeckel's law than not?), only in reverse order. Such a deep alkalinization of internal environment caused assembly of genetic material and former viral bodies into a single structure (later chromosome), as it was before after leaving of the reproduced parts of the virus from acidified zone external metabolism of vesicles into a neutral aqueous medium of archaic ocean. Further association of the said assembly with lipids of inner side of a membrane corresponds to a passed stage of the interaction of polynucleotides with lipid layers era "began of began" prior to the viruses with open metabolism of "active" hydrocarbon droplets. Naturally, the subsequent acidification of the internal environment of vesicles, associated with the beginning of recycling material depot, at first launched a replication of data connections, and then, with increasing acidity of the medium, the reproduction of other compounds involved in metabolism. Do not dwell on the analysis of the mass of errors associated with development in the processes of evolutionary transformation of specific mechanisms to regulate the speeds and depth of acidification of the internal environment. Note that exactly such mechanisms using acidification of medium as a key factor in their action, eventually, provided not only the completeness of the doubling of all the compounds involved in the internal metabolism, but division of vesicles into two parts with a full value metabolism, i.e. their transformation into the first cells.

Let us explain the last statement. With the gradual acidification of the medium changes the ability of compounds to associating with lipids. Some of them are accumulated on the inner surface of the membrane, while others can go into the aqueous environment. Changing the number of organics on lipids entails not only a change in internal energy of the membrane, but may accompanied and by changes in the shape of the vesicle. In the process of evolutionary selection were chosen corresponding compounds, synthesized in vesicles, and the mode of acidification of the internal environment, which provided the appearance of the constrictions and the collapse of dividing vesicles into two parts. In this way, in our opinion, formed from vesicles the first cells with anaerobic archaic metabolism, already capable, albeit with errors, reproduce oneself entirely. They gave rise to the development 
of cells, which form in the cytoplasm the depot of energy-intensive material necessary for division. These first cells having only the rudiments of the cytoskeleton could not adequately withstand the aggressive action of the water element and were perishing. Their contents turned out to the common heritage of the emerging living world. However, the speed of their reproduction at abundance of energy-intensive and plastic material (transition of organic from the atmosphere into the aqueous medium is not over), eventually had higher the rate of their ruin. "First-burning" of cells was held, was launched development of anaerobes, and not with a single cell, which could perish along with the offspring, but in mass quantity, that eliminates accidental.

\section{FINAL STAGE OF ABIOGENESIS}

This stage is characterize not only by the further development of oxygen-free metabolism of first cells and "active" vesicles, but also and by new phenomenon - the emergence of photosynthesis. In the period under review the development of abiogenesis in the aquatic environment inside and outside the vesicles with developed metabolism there were complex compounds that can store energy (eg macroergs) and transmit it to other compounds. Among them were also sensitive to sunlight, able to garner the energy and use it directly for the synthesis, or transmit it to other compounds. The atmosphere became more and more transparent that was conducive to photosynthesis. Formation free energy sources through the use of sunlight is made possible at a certain stage of cooling the Earth's surface, including water mediums. Such processes proceeded as in open aqueous medium, and within the vesicles, so how organics transition from the atmosphere into the aqueous medium is not yet ended and was continued the isolation of material of aqueous medium in vesicles by capturing necessary compounds at the closure of the lipid membranes. The latter circumstance in the future became a defining moment in the transformation of such vesicles in predecessors of proplastids and themselves plastids, in which occur general processes of metabolism.

An most important consequence of the emergence and rapid accumulation of vesicles, in metabolism of which was dominated the photosynthetic processes by the synthesis from carbon dioxide the carbon-containing compounds, became yield oxygen. Only after its accumulation in the atmosphere in sufficient amount became possible forming the aerobic metabolism in first cells.

It is believed that this accumulation of oxygen in the atmosphere is determined by the activities of prokaryotes with their ability to photosynthesize. In our analysis of the first in this case were the evolutionary precursors of plastids - vesicles with metabolism based on photosynthesis, because, in our opinion, prokaryotes else did not exist, because their predecessors - the vesicles with an external depot of the power-consuming and plastic material else has not "learned" to share.

Further development (complication) of vesicles was going in two main directions: in interacting with world of viruses and in interacting with each other. In a symbiotic information interaction with complicated world of viruses arosed the structures which provide for vesicles, firstly, greater stability in the turbulent environment, and, secondly, formation of energy metabolism, based on the use free oxygen in the oxidation processes. Of course, it is impossible to achieve absolute stability, so they are after their ruin "shared" by their content with new vesicles, since the phase of organics isolation was not over yet. Furthermore, the vesicles may adhere to each other, combining their contents.

One of the important factors that determined the further development of the living world became the ability to capture other vesicles or even cells at the formation of large vesicles. Huge biomass of all living organisms of the planet Earth originated from carbon-containing compounds, and passed, in our opinion, the vesicular stage. Therefore, the surface layers of the archaic ocean were filled mostly with vesicles with such density that such a possibility has been provided and a very long time. A detailed analysis of the separation processes of the developing biological material allows us to trace the sequence of formation of all major branches of cells: animal, plant and fungi, as well as prokaryotes. As part of a short article it is impossible to elaborate on such material. We note only that this approach 
finds its solution is also in the problem of initial accumulation of oxygen in the atmosphere as a consequence of long-term domination of vesicles with energetic metabolism on the base of photosynthesis. Grips at its birth by large vesicles of archaic cells with "active" vesicles and without them became the beginning of the formation of all types of cells. We emphasize that the specified "action" had the planetary scale, not as a separate act of "creation." Nature used the separation in time competing for energy and materials processes of formation of cellular structures (organelles) and the cells themselves of the above types. Precursors of mitochondria and nuclei (perhaps archaic cells) were formed and included in the structure of animal cells at a time when the oxygen pressure does not exceed 2 $\mathrm{mm} \mathrm{Hg}$. This is evidenced by homeostasis of the oxygen level in the cytoplasm of animal cells. In process of its grows in the aquatic environment, the predecessors of plastids have began gradually to displace predecessors mitochondria. Period from the beginning of this displacing to its completion - formation time of the fungal cells. At the final stage is setting a complete domination of predecessors plastid and are formed plant cells by the above scheme. Since the plastides and plant cells have been formed in the last turn, they included the achievements of previous stages of formation of the living world. On the one hand, the structural elements of precursors and the cells themselves and plants and fungi began more resistant to aggressive environment. Many elements and mechanisms of mitotic activity were "borrowed" from animal cells. On the other hand, they could not provide cytokinesis proper division of plant cells and fungal cells into two equivalent parts. Formation of the original fission mechanisms of these cells was carried out by the same principles of selection, that and of animal cells. With the accumulation in the depot of energy-intensive and plastic materials signal is forming for the start of its utilization, which is triggered by the achievement of a size sufficient to provision of energy of several cellular divisions. When reaching a certain level of acidification of the cytoplasm the material constriction or septum in prokaryotic cell and preprophase band and cell plate in the plant cells spontaneously is collected in these structures on the principle of minimum free energy of structure. The assembly of elements and material, as well as the order of percolation of processes during mitosis eventually became performed automatically, and the driving factor, as in the animal cell, became the cytoplasm acidification.

\section{CONCLUSION}

Formation of the complex and diverse mechanisms of cell activity and its reproduction began in an aqueous medium long before the appearance of the first cells in considered by us phase of abiogenesis in processes of interaction of the viral world with the world of lipid vesicles having initially anaerobic and, with the development, aerobic external and internal energetic metabolism. Leading link, a provider of new information, necessary for the evolutionary development of the living world were viruses. Short lifetime of vesicles due to the ongoing their birth inevitably was accompanied by the seizure with the water of the biological material that has provided a new flow of information as a necessary condition for the growth of populations of viruses and stability for the vesicles.

It is shown that the maximum possible power of groups metabolic processes competing for macroergs in vesicles was achieved thanks to the breeding of these groups in time. Means of this breeding becam the natural acidification of the internal environment by the synthesis macroergs, and the forcible her alkalinization by the their utilization for the needs of cell activity. Cyclical regimes of vesicles metabolism (and subsequently cells) were formed by selection of enzyme on activity of the catalysis of synthesis by higher values $\mathrm{pH}$, than catalysis of utilisation macroergs. The essential role in the appearance of the cells played selection of compounds by the type of symmetry (chirality) which extremely narrow the range of compounds that can participate in the internal metabolism of vesicles. This, in turn, ensured a successful search by trial and error of the respective forms of the compounds and mechanisms in reserved time for the nascent cellular world from the beginning of the transition of organics from the atmosphere to the 
surface of the planet and to completion of this transition.

At the initial stage of this period developed the anaerobic metabolism of vesicles. The logical conclusion of this development was the emergence of the first simplest anaerobic cells without chromosomes and nucleus (prokaryotes) that could reproduce itself.

Formation and development in some, else small part of vesicles and cells-anaerobes of the metabolism on the base of photosynthesis, provide gradual accumulation in the atmosphere of oxygen. This to ensure the formation and development in vesicles and first cells in addition to anaerobic energetic metabolism, that already existing, aerobic metabolism, which gave rise to the development of simplest (prokaryotic) cells aerobes.

One of the important factors that determined the development of the living world was the ability to capture by the formation of large vesicles other vesicles or even cells. To the moment of achievement in an atmosphere of oxygen partial pressure of $2 \mathrm{~mm} \mathrm{Hg}$, the captured "active" vesicles with preserved of the cyclicity of its aerobic metabolism gradually turned into the mitochondria, and cell-anaerobes - into the kernels, that can double their genetic material during mitosis than was launched start of the formation and evolutionary development of animal cells.

With further accumulation of oxygen in the aquatic environment has steadily increased the proportion of vesicles with the metabolism based on photosynthesis. Into such vesicles inevitably get elements of anaerobic and aerobic metabolisms. The isolation of these vesicles together with the vesicles-aerobic and anaerobic cells in the composition of a large vesicles gave rise to the formation and development of fungal cells. Captured vesicles transform into plastids and mitochondria, and cell - into nucleus.

Vesicles with complex metabolism comprising photosynthesis and utilisation of energy-intensive substrates by aerobic and anaerobic manner, in process of the accumulation of oxygen in the aqueous medium is practically superseded all other vesicles. Grips and the inclusion of such vesicles along with anaerobic cells as part of larger vesicles by the above given scheme, gave a start to the formation and development of plant cells. In these nascent cells there were no conditions for the formation of mitochondria, and the plastids were somewhat different from the plastid of fungal cell by versatility of their metabolism. So naturally turned out that many of the basic plant metabolic processes occur in plastids and in animals, these same processes are in the cytoplasm.

So ended the stage of abiogenesis - gradual complication inorganic substances before the biopolymers (nucleic acids, proteins, and others.), which are inherent in the basic properties of living things - metabolism, replication, flow homeostasis and selection of compounds on the type of symmetry, and the emergence of protocells - of descendants of hydrocarbonic aerosol of primary the atmosphere and of vesicles of the primary ocean.

\section{REFERENCES}

1. Zaritsky AR, Grachev VI, Vorontsov YuP, Pronin VS. Energy Aspects of Abiogenesis in the Atmosphere on Hydrocarbon Aerosol Nanodroplets. RENSIT, 2013, 5(2):105-125 (in Russ.).

2. Zaritsky AR, Vorontsov YuP, Pronin VS. Abiogenesis in the atmosphere: archaic metabolism, combinatorial replication and primary viruses. RENSIT, 2014, 6(1):110-123 (in Russ.).

3. Monin AS. Rannyaya geologicheskaya istoriya Zemli [Early geological history of the Earth]. Moscow, Nedra Publ., 1987, 261 p.

4. Gomes R, Levison HF, Tsiganis K, Morbidelli A. Origin of the Cataclysmic Late Heavy Bombardment Period of the Terrestrial Planets. Nature, 2005, 435(03676):466-469.

5. Hoyle F. Mathematics of Evolution. Acorn Enterprises LLC, Memphis, Tennessee, 1999, $142 \mathrm{p}$.

6. Oparin AI. The Origin of Life on Earth. NY, Academic Press, 1938.

7. Nelson DL, Cox MM. Lehninger Principles of Biochemistry. WH Freeman, 2012, 1100 p.

8. Zaritsky AR, Pronin VS. Biofizika osnovnykh rezhimov kletochnogo metabolizma. Funktsionalnye rezhimy kletki: sostoyanie pokoya 
i aktivnost' [Biophysics of cellular metabolism major modes. Functional modes cells: a state of rest and activity]. Bulletin of the Lebedev Physics Institute, 2006, 12:8-18 (in Russ.).

9. Zaritsky AR, Pronin VS. Biofizika osnovnykh rezhimov kletochnogo metabolizma. Rezhim deleniya kletki (mitoz). [Biophysics of cellular metabolism major modes. Mode of cell division (mitosis)]. Bulletin of the Lebedev Physics Institute, 2006, 12:19-27 (in Russ.).

10. Galimov EM. Fenomen zhizni [The phenomenon of life]. Moscow, URSS Publ., 2006, 254 p.

11. Fok MV, Zaritsky AR. Avtoregulyatsiya kak osnova gomeostaza kletok. [Autoregulation as a basis of cell homeostasis]. Moscow, Kosmoinform Publ., 1997, 122 p. 